with physical training the inevitable question arises as to whether meat is an essential constituent. There is no scientific evidence to show that meat is essential. The breakdown of the protein-containing tissues, particularly of muscle, during the performance of muscle work, provided there is an adequate supply of fat and carbohydrate or, in other words, of the energy-giving foodstuffs, is negligible. Yet if one examines the great majority of training diets meat is a prominent constituent. Practically all of the athletes at the Berlin Olympic games were large consumers of meat. Evidently the appetite or tradition or a real pica demands the presence of meat. Why this demand if there be no marked breakdown of muscle to repair? The explanation may in part lie in the fact that the extractives of meat are stimulating and have a definite and to many an attractive flavour. The presence of the flavour may help in the consumption of larger amounts of the, on the whole, flavourless fats and carbohydrates which are required for energy production. On the other hand, it must not be forgotten that strict vegetarians have shown themselves to be record-breakers in sport and to be capable of immense endurance. I think the conclusion to be reached must be that the presence of meat is not essential.

\section{FATS AND CARBOHYDRATES}

The protein problem has, it is true, aroused most discussion, but some very interesting facts are available regarding the consumption of fat. As long ago as 1866 Frankland had noted that men engaged in really laborious work consumed for preference fat-rich diets: "thick slices of bread surmounted with massive blocks of bacon in which mere streaks of lean were visible." Lavonius, who studied the food intake of professional Finnish wrestlers, found that calories derived from fat formed about 45 per cent. of the total intake, whereas those from protein only accounted for about 17 per cent. It has also been noted that in the diets of lumbermen and others engaged in hard work under severe environmental conditions the consumption of fat is high. If other lines regarding the need for fat be followed it is becoming increasingly evident that fat, or at least certain fatty acids, play a more important part than mere sources of energy.

There is no question about carbohydrate being the most readily available source of energy for the performance of muscle work. But, contrary to general belief, the recent work of Krogh et al. shows that there is no evidence during an hour's observation that glucose given during work, although it may cause a prompt rise in the blood-sugar level, well above resting level, is utilized. Apparently the carbohydrate has first to be converted into glycogen. Thus these authors maintain that in sporting events there ought to be about two days' rest before the contest, " to secure a complete filling up of the glycogen stores; and it appears that this cannot be done by taking large amounts of carbohydrate just prior to work."

I have cited some of the work relating to protein, fat, and carbohydrate simply as examples of the profundity of our ignorance of the whys and wherefores of somatic chemistry. If we know relatively little about the fundamental problem of the three energy-giving materials which have been intensively studied for many decades, we know considerably less, both quantitatively and qualitatively, about the action of mineral salts and vitamins.

\section{Diet Not the Only Factor in Malnutrition}

It may be thought that I am overstressing the negative side of the importance of food, that I am not giving it the importance which it so manifestly demands. If that be so I desire most categorically to deny the interpretation that I under-estimate its value. I have worked far too long at the problem. But I do most emphatically desire to bring home the point of view that diet alone is not the only factor involved in malnutrition. Far too many people take the path of least resistance. Seizing on the very fact that diet is important, they shut their eyes to all other possible factors or, if they do not wholly ignore them, they belittle them or do not explore their potentialities. There would possibly be no harm done if they stopped at the proclamation of their appreciation of an adequate dietary, but when they go on to promise all sorts of miracles which will happen if and when the average diet is altered in this way or that a very real danger arises. The majority of these miracles cannot and will not happen. The faith of the "man-inthe-street"-the man we all wish to help-in science and the scientific worker will be undermined.

\section{Conclusion}

We require to keep a broad and open mind on the problem of how best to achieve fitness. Just as there is no one cause of unfitness, so there is no one solution. It is a problem of many facets with as many lines of attack. It will not be achieved merely by the satisfaction of the somatic needs. Malnutrition of the spirit is quite as common as malnutrition of the body. The one reacts upon the other. In these days when the raucous enunciation of rights threatens to extinguish the still small voice of duty, it should not be forgotten that fitness demands discipline. Discipline is essential for right living. Right living means health. As Amiel said: "Health is the first of all liberties, and happiness gives us the energy which is the basis of health."

\section{SKIAGRAPHY OF THE CHEST *}

\section{BY}

\author{
GEORGE JESSEL, M.A., D.M., D.P.H.
}

Consultant Tuberculosis Officer, Lancashire County

Council; Visiting Medical Superintendent, Peel Hall Pulmonary Hospital

The use of skiagrams as an aid to diagnosis may well be regarded as the greatest advance in medicine during the present century, worthy to rank with the discovery of antiseptics and anaesthetics. Skiagraphy of the chest has become a most important and, indeed, an indispensable adjunct in diagnosis. Its routine application has often enabled a diagnosis of pulmonary disease to be made, even when the symptoms were referred to some other organ-for example, cases of so-called indigestion and gastritis or suspected appendicitis.

In the training of medical students it has long been customary to emphasize the importance of a careful examination of patients, based upon history, symptoms, and the routine use of the standard methods of physical examination. By such means the careful and experienced practitioner can accurately diagnose a fair proportion of the diseases of the chest from which his patients are suffering, while the consultant's wider experience of particular conditions will raise the standard of diagnosis still further. Indeed, some experienced physicians have in the past, by clinical methods alone, achieved a remarkable degree of accuracy in diagnosis. Their clinical instinct,

* Read in opening a discussion in the Section of Tuberculosis at the Annual Meeting of the British Medical Association, Plymouth, 1938. 
which we now know to be closely related to a thorough and careful detailed examination of each patient, coupled with an exceptional facility in recalling similar or related cases, has sometimes been little short of uncanny. Unfortunately the number of such gifted men at any one time is relatively few.

\section{Value of the Older Methods}

It is well known that more mistakes in diagnosis are die to failure to make a thorough examination of the patient than to lack of knowledge. The increasing popularity of skiagraphy, based on a growing appreciation of its value in the elucidation of difficult cases, ought not to permit any relaxation in the thoroughness with which the older methods of examination are employed. It is well known, as Sampson and Lawrason Brown (1931) and others have shown, that radiographs frequently reveal the existence of pulmonary disease in the apparent absence of any physical signs, even when the patient is carefully examined by an experienced physician, but there are likewise many cases where skiagraphic examination alone, in the absence of information derived from a careful history and physical examination, may provide information that is equivocal or misleading. Accurate diagnosis depends upon a careful appraisement of all the available evidence, including history and clinical, laboratory, and radiological examinations. Skiagraphy should be regarded as an additional weapon in our diagnostic armoury, but we cannot afford to ignore the information derived from interrogation, eye, hand, and ear. As Sir Arthur MacNalty (1937) has recently said, "pulmonary tuberculosis still reveals itself by physical signs and symptoms; in other words, inspection, palpation, percussion, and auscultation are not displaced, but reinforced, by a skiagram of the chest."

\section{The Development of Skiagraphy}

The National Health Insurance Act had as one of its objects the encouragement of insured persons to consult a doctor at an early stage of their illness, and thus overcome the natural tendency of the sick to defer medical examination until symptoms became too obvious or painful to be further ignored. The vast increase in the amount of work thus thrown upon the busy doctor has not infrequently rendered a thorough and detailed examination impossible. Furthermore, recent advances in medical knowledge have placed increasing responsibilities upon the overworked practitioner. Often some method was needed that would furnish greater precision in diagnosis than could be provided by ordinary physical examination alone and at the same time introduce some objective means of checking conclusions previously reached. The development of skiagraphy was thus timely. In particular, its use in doubtful chest conditions, especially in the diagnosis of pulmonary tuberculosis, has been of incalculable value. It is, unfortunately, true that progress has been slow and uneven. Indeed, until well after the great war the use of skiagrams, even by the enlightened, was irregular and spasmodic; in large centres patients were sometimes sent to radiologists attached to general hospitals, but elsewhere little or nothing in this direction was practicable. Those were the days when an $x$-ray diagnosis of "hilum tuberculosis" or "peribronchial fibrosis" was fashionable. Improvements in technique and increased experience have resulted in a reliable standard of diagnosis by competent radiologists, and ample facilities for the production of first-class skiagrams of the chest have existed in many areas for a number of years. Unfortunately, however, outside the big centres, and especially in the smaller urban and rural districts, these facilities are still patchy and irregular. It is the duty of the councils of counties and county boroughs to make provision for the specialist diagnosis of tuberculosis available for the whole population, but some local authorities are still laggards in this respect. It is thus not surprising that even in 1937 pleas were still being made for an increase in the use of skiagrams in the diagnosis of chest diseases in general and of tuberculosis in particular (Davies, 1937). On the other hand, although there is good reason to believe that a steadily increasing number of medical practitioners realize the value and importance of skiagraphy, there are still far too many who fail to make enough use of the facilities now available.

\section{Its Use in Diagnosis of Pulmonary Tuberculosis}

It is arguable that skiagraphy of the chest need only be employed on occasions, more or less frequent, depending upon the personal factor, when the physician finds that ordinary clinical methods are insufficient to enable him to make a firm diagnosis. There are, it is true, a number of well-marked cases of pulmonary tuberculosis, with tubercle bacilli in the sputum, where $x$-ray examination is unnecessary. On the other hand, there are a large number of cases where, owing to the failure to make use of skiagraphy, early tuberculosis has been missed until the disease has become evident even to the laity.

\section{In 1933 I wrote as follows (Jessel, 1933):}

"Diagnosis has of recent years become much more precise as the result of the intelligent use of good $x$-ray apparatus. In cases of suspected fracture failure to use such facilities ranks as negligence, and I think the time is not far distant when a similar view will be taken in cases where there are reasonable grounds for suspecting tuberculosis."

This view has received striking confirmation by a recent decision of the Court of Appeal (Connolly v. Rubra). According to the reports of the case Lord Justice Greer (1937) remarked :

"Tuberculosis is one of many diseases with which a general practitioner commonly has to deal. If the doctor has any doubt whether or no there are signs of tuberculosis, it is his obvious duty to make further examination to resolve that doubt and to make it clear that nothing further could be done for the patient. A doctor who is not over-confident of his own judgment will, when he finds himself in a difficulty, call an expert and decide according to the expert's advice. To enable the expert's opinon to be of value it is not enough to do what Dr. Rubra did at a later date-namely, to take one specimen of sputum and be content with that if the result is negative; he must take many specimens of sputum and, in addition, he ought to have an $x$-ray examination and to watch the patient to see whether he is progressing or going back as time goes on."

The disadvantages resulting from delay in the use of skiagraphy are (1) the longer time that is usually needed to reach a diagnosis, and (2) the tendency to play for safety and to diagnose a non-existent tuberculosis. I refer specifically to tuberculosis because, being an infectious disease, it is most important that it should be diagnosed as early as possible in order that remedial and preventive measures may be taken without delay. Pulmonary tuberculosis should be regarded as at least a possibility and skiagraphy be employed in the following circumstances:

1. In cases provisionally diagnosed as bronchitis or "bronchial catarrh" which do not clear up within four weeks.

2. Where patients complain of indigestion and dyspepsia. Many so-called "gastric or duodenal ulcers" are really manifestations of tuberculous toxaemia. 
3. In cases of alleged haemoptysis.

4. In young persons with symptoms of debility or anaemia.

5. In cases of chronic hoarseness.

6. In cases of ischio-rectal abscess.

7. In all cases where there is a clear history of tuberculosis in some member of the immediate family or household.

8. Where there is a history of some other close association with a case of pulmonary tuberculosis-for example, friend or workmate in office or factory.

9. Where a case of tuberculous meningitis or other form of non-pulmonary tuberculosis is found, attention should be directed to adult members of the household, even if stated to be in good health, in order to discover a possible source of infection.

When a diagnosis of pulmonary tuberculosis has been made skiagraphy is often of great value:

1. To determine the extent and nature of the diseasefor example, unilateral or bilateral, presence of cavities, fibrosis, calcification, etc. The information thereby obtained is often of great assistance in deciding the nature of treatment.

2. During the course of treatment serial skiagraphy is essential in artificial pneumothorax and whenever surgical measures are contemplated-for example, phrenic operations, division of adhesions, and thoracoplasty.

3. Serial skiagraphy also gives great service in enabling the progress of the disease to he readily ascertained, as well as in providing opportunities for the study of "living pathology."

\section{Skiagraphy in Differential Diagnosis}

Apart from the question of tuberculosis, skiagraphy of the chest is important in the differentiation of pulmonary disease. Assuming that tuberculosis can be definitely excluded, skiagraphy may clearly indicate or suggest chronic bronchitis, bronchiectasis (especially when used in conjunction with lipiodol), pleurisy, pneumonias, abscess, malignant disease, silicosis. Unsuspected morbus cordis may likewise be discovered. Caution must, however, be exercised in the interpretation of skiagrams, as the appearances-for example, in cancer, tuberculosis, or silicosis-may at times be indistinguishable. An opacity may be due to fluid, thickened pleura, consolidation, atelectasis, abscess, or underlying new growth. Consistently accurate diagnosis is only possible when skiagraphy is correlated with clinical evidence. It demands judgment and experience. Indeed, skiagraphy may easily lead to misunderstanding and confusion: shadows of bronchi and blood vessels seen in their various aspects and angles may be misinterpreted as evidence of disease. It is not impossible by skiagraphy, as by older methods of clinical examination, to diagnose non-existent tuberculosis, which is almost as serious as failing to detect tuberculosis when it is present.

Skiagraphy of the chest is a specialty within a specialty. The taking of a good skiagram and its interpretation are by no means easy. The well-to-do are, in general, adequately catered for in this respect by radiologists, and there are also a few consultants and general practitioners who can produce excellent skiagrams. The cost, however, is undoubtedly a deterrent; but fortunately skiagraphy is obtainable at or in connexion with many tuberculosis dispensaries and the chest clinics attached to some large hospitals. This provision needs to be extended so as to make chest skiagraphy readily available for every citizen. The presence of $x$-ray apparatus at or in close association with a tuberculosis dispensary or chest clinic affords an ideal solution of the problem.

\section{Routine in a Lancashire Area}

This is not the occasion to deal with technical details, which are mainly of interest to those practising radiology.
It is, however, desirable to illustrate what has been said above by a brief description of what obtains in the Lancashire area with which I am associated. The population of the area is abnut 370,000 , and is mainly urban in character. It contains five tuberculosis dispensaries, as well as a small hospital of fifty-seven beds for adult male pulmonary cases. The medical staff consists of three tuberculosis officers working as a team, one of whom (in this case myself) acts as radiologist at the central dispensary, where the $x$-ray apparatus is situated. Early in $1923 \mathrm{I}$ used a gas-tube apparatus, but since 1932 an all-British singlevalve unit that has given complete satisfaction. Exposures of one-tenth of a second at four feet distance have been found to give good results. Two afternoon sessions are held weekly. Nearly all the patients, who are referred to the dispensaries or are seen at home in consultation with the insurance practitioner or private doctor, attend one of these sessions by appointment. Bed cases, if not too ill, are brought by ambulance, and by this means acute non-tuberculous conditions can be identified and studied. On the same afternoons an artificial pneumothorax refill clinic is held elsewhere in the building by another tuberculosis officer, and these patients are screened at the beginning of the session. Screenings for diagnosis are seldom done, as the information to be derived from them is regarded as of far less value than a skiagram, which provides a permanent record to be examined and discussed at any subsequent time. In difficult cases, oblique and lateral, also postero-anterior flat films are regularly taken. Non-pulmonary as well as pulmonary cases are dealt with, and the total volume of work done may be gauged by the fact that 1,339 skiagrams were taken and 557 screenings done during 1937. It is necessary to emphasize the point that the skiagrams are all taken by a tuberculosis officer, who has before him the clinical notes made by himself or by one of his two colleagues. During each $x$-ray session two medical officers work in conjunction, one as a clinician radiologist, the other giving useful help in chest, throat, and other examinations. Cases definitely tuberculous are dealt with at once, the patient interviewed, and the necessary treatment arranged. Other patients are referred to the dispensary nearest their homes Ior re-examination at a later date, but most cases are definitely diagnosable by this combined clinico-radiological method within a fortnight. On the morning following each $x$-ray session the three tuberculosis officers meet as a board to examine the films, together with the clinical dossier of each case. The position is analogous to that of a bench of magistrates, weighing the whole of the available evidence derived from various forms of examination, including skiagraphy, and reaching a conclusion based upon the different findings taken separately and in conjunction. It is obviously advantageous to be able to bring the clinician and the radiologist so closely together, especially when it is noted that even expert whole-time radiologists admit that by skiagraphy alone the differential diagnosis of pulmonary conditions is often impossible; indeed, the number of such cases has been placed as high as 40 per cent. Moreover, a radiologist who has no clinical experience or is not regularly engaged in clinical work is often greatly handicapped in endeavouring to interpret what he sees. This disadvantage can, however, be overcome to some extent if the radiologist and clinician actually meet and discuss cases of special difficulty or interest.

Finally, as soon as a diagnosis is made a report is sent to the patient's doctor, and, if of sufficient interest, a few days later a reduced print of the skiagram. There is reason to believe that these prints are appreciated and 
help to encourage the use of our radiological clinic. This, in brief, illustrates the procedure $I$ have adopted for over fifteen years.

\section{Conclusion}

Medical practitioners are gradually recognizing the help that chest skiagraphy can give, but they sometimes have difficulty in obtaining a patient's consent to undergo examination. This reluctance is usually related to the popular idea that tuberculosis is necessarily incurable. The wise and tactful practitioner will explain that the object of skiagraphy is to make sure that the patient is all right, or at least only suffering from disease in an early stage. Driving and other tests are becoming more common among the general public, and a few minutes' simple explanation will often reassure a timid patient.

Skiagraphy of the chest is so important for accurate diagnosis that it can seldom be omitted with safety. It is the most valuable single factor in the diagnosis of pulmonary disease.

REFERENCES

Davies, G. Ivor (1937). Tubercle, 18, No. 11, 482

Greer, Lord Justice (1937). Lancet, 1, 1006.

Jessel, G. (1933). Lancs County Council, Annual Report of Central Tuberculosis Officer, p. 88.

MacNalty, Sir Arthur (1937). Brit. J. Tuberc., 31, 126.

Sampson, H. L., and Brown, L. (1931). Amer. J. Roentgen., 25, 209.

\section{THREE CASES OF CL.WELCHII INFECTION FOLLOWING ABORTION}

BY

\author{
R. Y. DAWBARN, M.D., \\ Pathologist, Walton Hospital
}

AND

BRYAN WILLIAMS, M.D., M.C.O.G., F.R.C.S.Ed. Senior Assistant Medical Officer, Walton Hospital

This condition appears to be uncommon, or at any rate infrequently recognized, and we hope, therefore, that it may be of value to record three more cases. In his very thorough review of the whole question of post-abortal and puerperal gas gangrene, Hill (1936) was able to find only eighty-four cases described in the literature, to which he added a further series of thirty cases of his own.

The interest to us has been twofold: first, as regards the differential diagnosis, and, secondly, by reason of the rapidly fatal course of the illness. In both of the cases of septicaemia the onset was so sudden and the manifestations were so dramatic that chemical poisoning was suspected. These suspicions were strengthened by the fact that both patients had been taking large doses of pills, presumably with the object of procuring abortion. We record the more recent of these two cases first because the diagnosis was confirmed bacteriologically. The other case occurred in 1934, and though we feel sure now of the diagnosis, bacteriological confirmation is lacking. The third case also ran a fulminating course, although the clinical picture was different and the diagnosis was made soon after the admission of the patient to hospital.

\section{Case I}

A married woman aged 30 was admitted to Walton Hospital at 2.30 p.m. on December 13, 1937. Her three previous pregnancies had been normal. Her last menstrual period was on October 20. During the previous week she had been taking pills of iron and aloes, twenty in all. At 2 a.m. on the day of admission she was seized with severe lower abdominal pain and vomiting. At 11 a.m. there was considerable vaginal bleeding.

On admission the patient showed signs of severe shock, and her pulse was feeble. Her temperature was $97^{\circ}$, pulse 96, and respirations 26 . The lower abdomen was extremely tender and rigid. There was slight vaginal bleeding, and on examination the cervix was closed and the uterus enlarged to about the size of a sixteen-weeks pregnancy. There was general pelvic tenderness. A provisional diagnosis of either septic abortion with pelvic peritonitis or of ectopic pregnancy was made.

At 6 p.m. her general condition appeared to have improved slightly. The lower abdomen and face had become flushed. About $2 \mathrm{c.cm}$. of thick dark brown urine was withdrawn by a catheter; microscopically, this showed no intact red blood cells. By 8 p.m., however, her condition had become much worse. There was now an extensive bluish flush on the abdomen and face. On vaginal examination the uterus seemed to be larger. Further catheterization produced only a few drops of almost black urine. Blood taken from a vein showed evidence of haemolysis, the plasma being the colour of burgundy. Death occurred at 9.10 p.m., or within seven hours of admission, and just over nineteen hours after the illness began. Shortly before death a crackling sensation was elicited on palpation of the lower abdomen.

\section{POST-MORTEM EXAMINATION}

The surface of the body showed a dusky bronzed hue, most marked on the face and abdomen. The deeper parts were very dark, and slight crepitation was noticed on cutting through the tissues of the neck and uterus. The lungs were very congested. There was haemolytic staining of the blood vessels and endocardium. The kidneys, weighing 6 and $6 \frac{1}{2} \mathrm{oz}$., were almost black. The spleen was about twice the normal size, and was tense and nearly black. Frothy fluid exuded from the cut surface. The liver showed cloudy swelling with a little gas under the capsule. The stomach revealed no sign of chemical poisoning. The intestines were pink and were not ulcerated. The uterus was greyish black, $4 \frac{1}{2}$ by $3 \frac{1}{2}$ inches, and very slightly crepitant. A little blood and necrotic material was found in the uterus, and was kept for section. No evidence of mechanical injury was found. The peritoneum contained dark-coloured fluid. A corpus luteum was present in the right ovary.

Films made directly from the peritoneal fluid and splenic puip showed great numbers of Gram-positive bacilli resembling $\mathrm{Cl}$. Welchii. Cultures were made from the spleen anaerobically, and a good growth of $\mathrm{Cl}$. welchii was obtained, which gave a characteristic stormy reaction in milk after incubation overnight.

Sections were cut of the uterus and its contents, the liver, and kidney, and were stained by haemalum and eosin and by Gram's method. The uterine contents consisted of necrotic decidua, blood, many Gram-positive bacilli, and other organisms. No placental villi were found. The uterine wall showed evidence of infection, and there were many dilated vessels containing infected clot. The muscle took the stain badly, and parts appeared to be almost necrotic. Numerous Gram-positive bacilli were seen throughout, especially in the blood vessels. The appearance of the kidney was suggestive of advanced post-mortem change, although the necropsy was performed well within twenty-four hours of death. In some areas the nuclei remained quite unstained, and there was marked degeneration of the glomeruli and cells lining the tubules. In addition to this many of the tubules were filled with brownish material which was evidently derived from the blood as a result of the haemolysis. The liver cells showed extreme degeneration, in many parts amounting to necrosis. The stain for organisms showed Gram-positive bacilli in both liver and kidney.

\section{Case II}

The following notes are taken from the records of Walton Hospital. One of us saw this patient before she died, and 